\title{
Numerical experiments modelling turbulent flows
}

\author{
Jiří Trefilík ${ }^{1, a}$, Karel Kozel ${ }^{1,2, b}$, and Jaromír Příhoda ${ }^{1, c}$ \\ 1 Institute of Thermomechanics AS CR, v. v. i. \\ 2 Faculty of Mechanical Engineering, CVUT Prague
}

\begin{abstract}
The work aims at investigation of the possibilities of modelling transonic flows mainly in external aerodynamics. New results are presented and compared with reference data and previously achieved results. For the turbulent flow simulations two modifications of the basic $k-\omega$ model are employed: SST and TNT. The numerical solution was achieved by using the MacCormack scheme on structured non-ortogonal grids. Artificial dissipation was added to improve the numerical stability.
\end{abstract}

\section{Mathematical models}

\subsection{Navier-Stokes equations}

The two-dimensional laminar flow of a viscous compressible liquid is described by the system of Navier-Stokes equations

$$
W_{t}+F_{x}+G_{y}=R_{x}+S_{y}
$$

where

$$
W=\left(\begin{array}{c}
\rho \\
\rho u \\
\rho v \\
e
\end{array}\right), F=\left(\begin{array}{c}
\rho u \\
\rho u^{2}+p \\
\rho u v \\
(e+p) u
\end{array}\right), G=\left(\begin{array}{c}
\rho v \\
\rho u v \\
\rho v^{2}+p \\
(e+p) v
\end{array}\right)
$$

and

$$
R=\left(\begin{array}{c}
0 \\
\tau_{x x} \\
\tau_{x y} \\
u \tau_{x x}+v \tau_{x y}+\lambda T_{x}
\end{array}\right), S=\left(\begin{array}{c}
0 \\
\tau_{x y} \\
\tau_{y y} \\
u \tau_{x y}+v \tau_{y y}+\lambda T_{y}
\end{array}\right)
$$

with shear stresses given for the laminar flow by equations

$$
\tau_{x x}=\frac{2}{3} \eta\left(2 u_{x}-v_{y}\right), \tau_{x y}=\eta\left(u_{y}+v_{x}\right), \tau_{y y}=\frac{2}{3} \eta\left(-u_{x}+2 v_{y}\right) .
$$

This system is enclosed by the equation of state

$$
p=(\kappa-1)\left[e-\frac{1}{2} \rho\left(u^{2}+v^{2}\right)\right] \text {. }
$$

In the above given equations, $\rho$ denotes density, $u, v$ are components of velocity in the direction of axis $x, y, p$ is pressure, $e$ is total energy per a unit volume, $T$ is temperature, $\eta$ is dynamical viscosity and $\lambda$ is thermal conductivity coefficient. The parameter $\kappa=1.4$ is the adiabatic exponent.

\footnotetext{
a e-mail: jiri.trefilik@fs.cvut.cz

b e-mail: karel.kozel@fs.cvut.cz

c e-mail: prihoda@it.cas.cz
}

\subsection{Reynolds averaged Navier-Stokes equations}

For the modelling of a turbulent flow, the system of RANS (Reynolds Averaged Navier-Stokes) equations enclosed by a turbulence model is used. Two different turbulence models with the turbulent viscosity were tested, one algebraic, Baldwin-Lomax and the two-equation $k-\omega$ model according to Wilcox. The system of averaged Navier-Stokes equations is formally the same as (1), but this time the flow parameters represent only mean values in the Favre sense, see [3]. The shear stresses are given for the turbulent flows by equations

$$
\begin{aligned}
\tau_{x x} & =\frac{2}{3}\left(\eta+\eta_{t}\right)\left(2 u_{x}-v_{y}\right), \\
\tau_{x y} & =\left(\eta+\eta_{t}\right)\left(u_{y}+v_{x}\right), \\
\tau_{y y} & =\frac{2}{3}\left(\eta+\eta_{t}\right)\left(-u_{x}+2 v_{y}\right),
\end{aligned}
$$

where $\eta_{t}$ denotes the turbulent dynamic viscosity according to the Boussinesq hypothesis. The Reynolds number is defined by $R e=\frac{u_{\infty} L}{\eta_{\infty}}$ and the Mach number by $M=\sqrt{\frac{q}{a}}$ where $q=\sqrt{\left(u^{2}+v^{2}\right)}$ and $a$ is the local speed of sound.

All the computations were carried out using dimensionless variables with reference variables given by inflow values. The reference length $L$ is given by the width of the computational domain.

\section{Turbulence models}

\subsection{TNT model}

So called TNT model is a modification of the classical $k-\omega$ model in which the turbulent viscosity is given as

$$
\eta_{t}=\rho \frac{k}{\omega} .
$$

$k$ denotes turbulent energy and $\omega$ is a specific dissipation rate. Values of $k$ and $\omega$ are obtained as a solution of equations 


$$
\begin{gathered}
\frac{\partial(\rho k)}{\partial t}+\frac{\partial\left(\rho u_{j} k\right)}{\partial x_{j}}=P_{k}+\frac{\partial}{\partial x_{j}}\left[\left(\mu+\sigma_{k} \mu_{t}\right) \frac{\partial k}{\partial x_{j}}\right]-\beta^{*} \rho k \omega \\
\frac{\partial(\rho \omega)}{\partial t}+\frac{\partial\left(\rho u_{j} \omega\right)}{\partial x_{j}}=\alpha \frac{\omega}{k} P_{k}+\frac{\partial}{\partial x_{j}}\left[\left(\mu+\sigma_{\omega} \mu_{t}\right) \frac{\partial \omega}{\partial x_{j}}\right]-\beta \rho k \omega^{2}+C_{D}
\end{gathered}
$$

where $P_{k}=\tau_{i j} \partial u_{i} / \partial x_{j}$ represents the production of turbulent energy,

$$
C_{D}=\frac{\rho}{2 \omega} \max \left(\frac{\partial k}{\partial x_{i}} \frac{\partial \omega}{\partial x_{i}}, 0\right)
$$

and $\alpha=0.553, \beta=\frac{3}{40}, \beta^{*}=\frac{9}{100}, \sigma_{\omega}=0.5, \sigma_{k}=0.666$.

\section{$2.2 S S T$ model}

$S S T$ model is another modification of $k-\omega$ model:

$$
\begin{aligned}
\frac{\partial}{\partial t}(\bar{\rho} k)+\frac{\partial}{\partial x_{j}}\left(\bar{\rho} \tilde{U}_{j} k\right)= & P_{k}+\frac{\partial}{\partial x_{j}}\left[\left(\mu+\sigma^{*} \mu_{t}\right) \frac{\partial k}{\partial x_{j}}\right]-\beta^{*} \bar{\rho} k \omega \\
\frac{\partial}{\partial t}(\bar{\rho} \omega)+\frac{\partial}{\partial x_{j}}\left(\rho \tilde{U}_{j} \omega\right) & =\gamma \frac{\omega}{k} P_{k}+\frac{\partial}{\partial x_{j}}\left[\left(\mu+\sigma \mu_{t}\right) \frac{\partial \omega}{\partial x_{j}}\right](12) \\
& -\beta \bar{\rho} \omega^{2}+2 \bar{\rho}\left(1-F_{1}\right) \sigma_{2} \frac{1}{\omega} \frac{\partial k}{x_{j}} \frac{\partial \omega}{x_{j}}
\end{aligned}
$$

The last term in the second equation expresses lateral diffusion. Turbulent viscosity is given by the formula

$$
v_{t}=\min \left(\frac{k}{\omega}, \frac{a_{1} k}{F_{2} \Omega}\right)
$$

and the functions $F_{1}$ a $F_{2}$ are defined as follows:

$$
\begin{gathered}
F_{1}=\tanh \left(\Gamma_{1}^{4}\right), \\
\Gamma_{1}=\min \left[\max \left(\frac{\sqrt{k}}{\beta^{*} \omega y} ; \frac{500 v}{\omega y^{2}}\right) ; \frac{4 \bar{\rho} \sigma_{2} k}{D_{\omega} y^{2}}\right],
\end{gathered}
$$

where $y$ is the distance from the wall

$$
\begin{gathered}
D_{\omega}=\max \left(2 \bar{\rho} \sigma_{2} \frac{1}{\omega} \frac{\partial k}{x_{j}} \frac{\partial \omega}{x_{j}} ; 10^{-20}\right), \\
F_{2}=\tanh \left(\Gamma_{2}^{2}\right),
\end{gathered}
$$

and

$$
\Gamma_{2}=\max \left(\frac{\sqrt{2 k}}{0.09 \omega y} ; \frac{500 v}{\omega y^{2}}\right) .
$$

Model's constants are given by the formula

$$
\phi=F_{1} \phi_{1}+\left(1-F_{1}\right) \phi_{2},
$$

where

$$
\kappa=0.41, \beta^{*}=9 / 100, \sigma^{*}=0.85, a_{1}=0.31,
$$

$\beta_{1}=3 / 40, \sigma_{1}=1 / 2, \sigma_{1}^{*}=1 / 2, \gamma_{1}=\beta_{1} / \beta^{*}-\sigma_{1} \kappa^{2} / \sqrt{\beta^{*}}$,

and

$\beta_{2}=0.0828, \sigma_{2}=0.85, \sigma_{2}^{*}=1, \gamma_{2}=\beta_{2} / \beta^{*}-\sigma_{2} \kappa^{2} / \sqrt{\beta^{*}}$.

\section{Boundary conditions for $k$ and $\omega$ :}

- Inlet:

- $k_{\infty}=\frac{3}{2}(0.001 \bar{U})^{2}$.

$-\omega_{\infty}=90 \frac{k_{\infty}}{v}$

- Wall:

$-k=0$.

- $\omega$ asymptotically approaches $+\infty$, very high value prescribed.

- Outlet: $k$ and $\omega$ extrapolated from the flow.

\section{Numerical methods}

For the modelling of the flow cases Lax-Wendroff finite volume method scheme was used on non-orthogonal structured grids of quadrilateral and hexahedral cells $D_{i j(k)}$.

- Predictor step:

$$
W_{i, j}^{n+1 / 2}=W_{i, j}^{n}-\frac{\Delta t}{\mu_{i, j}} \sum_{k=1}^{4}\left[\left(\tilde{F}_{k}^{n}-\frac{1}{R e} R_{k}^{n}\right) \Delta y_{k}-\left(\tilde{G}_{k}^{n}-\frac{1}{R e} S_{k}^{n}\right) \Delta x_{k}\right] .
$$

- Corrector step:

$$
\begin{aligned}
W_{i, j}^{n+1}= & \frac{1}{2}\left(W_{i, j}^{n}+W_{i, j}^{n+1 / 2}\right)-\frac{\Delta t}{2 \mu_{i, j}} \sum_{k=1}^{4}\left[\left(\tilde{F}_{k}^{n+1 / 2}-\frac{1}{R e} R_{k}^{n+1 / 2}\right) \Delta y_{k}\right. \\
& \left.-\left(\tilde{G}_{k}^{n+1 / 2}-\frac{1}{R e} S_{k}^{n+1 / 2}\right) \Delta x_{k}\right]+A D\left(W_{i, j}^{n}\right) .
\end{aligned}
$$

The Mac Cormack scheme in the cell centered form was applied to solving the system of RANS equations. Convective terms $F, G$ are considered in predictor step in forward form and in the corrector step in upwind form of the first order of accuracy, dissipative terms in central form of the second order of accuracy. To indicate this we denote their numerical approximation as $\tilde{F}, \tilde{G}$.

The scheme was extended to include Jameson's artificial dissipation because of the stability of the method

$$
\begin{aligned}
A D\left(W_{i, j}^{n}\right) & =C_{1} \psi_{1}\left(W_{i-1, j}^{n}-2 W_{i, j}^{n}+W_{i+1, j}^{n}\right) \\
& +C_{2} \psi_{2}\left(W_{i, j-1}^{n}-2 W_{i, j}^{n}+W_{i, j+1}^{n}\right),
\end{aligned}
$$

where

$$
\begin{aligned}
& \psi_{1}=\frac{\left|p_{i-1, j}^{n}-2 p_{i, j}^{n}+p_{i+1, j}^{n}\right|}{\left|p_{i-1, j}^{n}\right|+\left|p_{i, j}^{n}\right|+\left|p_{i+1, j}^{n}\right|}, \\
& \psi_{2}=\frac{\left|p_{i, j-1}^{n}-2 p_{i, j}^{n}+p_{i, j+1}^{n}\right|}{\left|p_{i, j-1}^{n}\right|+\left|p_{i, j}^{n}\right|+\left|p_{i, j+1}^{n}\right|} .
\end{aligned}
$$




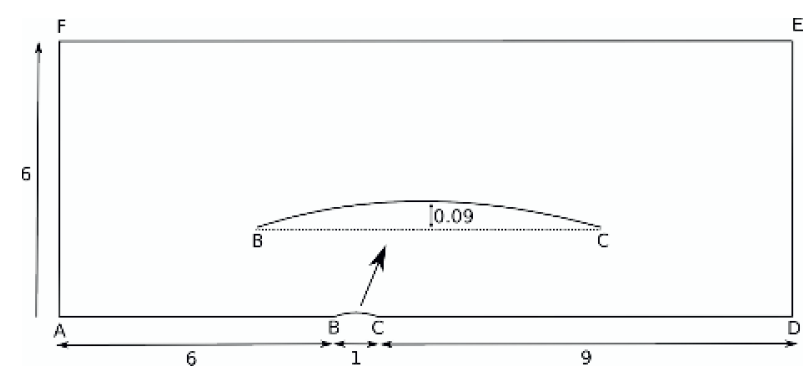

Fig. 1. Computational domain

The convergence to the steady state is followed by log $L_{2}$ residual defined by

$$
\operatorname{Res} W^{n}=\sqrt{\frac{1}{M} \sum_{k}\left(\frac{W_{k}^{n+1}-W_{k}^{n}}{\Delta t}\right)}
$$

where $M$ is a number of all cells in the computational domain.

\section{Formulation of the problem}

The computational domain is demonstrated on the picture (1). The line AF designates the inlet boundary, DE is the outlet, BC is the wall and the rest is defined as symmetric boundary.

\section{Results}

The figure (2) represents the result of simulation obtained with the $S S T$ model for the same parameters as those of the reference result (5). The agreement is very good and the symmetric simulation (4) indicates that small perturbations do not spread in the calculation domain which would destroy the symmetry. Figure (3) shows similar result obtained previously with the usage of the TNT modification of $k-\omega$ model. It can be noted that the $S S T$ simulation leads to developement of a smaller wake and also the maximum value of velocity that exists in the flow field is significantly higher (around 1.32) compared to the one in the TNT model simulation ( around 1.26).

\section{Conclusion}

A new method for the simulation of turbulent transonic flows has been developed and tested. The results obtained appear to be more realistic compared to those of the $T N T$ method. At the same time the implementation of the $S S T$ method is considerably more complex and therefore more error prone. The method is also approximately $25 \%$ slower in terms of the time needed to perform the calculation. Further simulations especially for the internal aerodynamics will be carried out to able to better assess its characteristics.

\section{Acknowledgment}

The work was partly supported by by the institutional support RVO 61388998 and by the grant projects GA AS CR IAA 200760 81, GA CR P101/10/1329, P 101/12/1271 and SGS 10/243/OHK2/3T/12.

\section{References}

1. R. Dvořák, Transonic Flows, Academia, Prague (1986, in Czech).

2. R. Dvořák, K. Kozel, Mathematical modelling in aerodynamics, CTU in Prague, Prague (1996, in Czech)

3. A. Favre, Jour. de Mecanique, 4, 361 (1965)

4. M. Feistauer, J. Felcman, I. Straškraba, Mathematical and Computational Methods for Compressible Flow, Oxford University Press (2003)

5. A. Hellsten, AIAA J., 36, 2528 (1998)

6. A. Hellsten, AIAA J., 43, 1857 (2005)

7. C. Hirsch, Numerical Computation of Internal and External Flows, Volume II, - Computational Methods for Inviscid and Viscous Flows, John Willey\&Sons (1990)

8. J. Holman, J. Furst, Proceedings: Colloquium Fluid Dynamics 2008, 11, Institute of Thermomechanics, AS CR, v. v. i., Prague (2008)

9. J. Huml, J. Furst, K. Kozel, J. Příhoda, Proceedings: Topical Problems of Fluid Dynamics 2009, 45 (2009, in Czech).

10. J. Huml, J. Holman, J. Furst, K. Kozel, Proceedings: Topical Problems of Fluid Dynamics 2010, 73 (2010)

11. J.C. Kok, AIAA J., 38, 1292 (2000)

12. F.R. Menter, AIAA J., 30, 1657 (1992)

13. F.R. Menter, AIAA J., 32, 1598 (1994)

14. P. Pořízková, Numerical Solution of Compressible Flows Using Finite Volume Method, PhD Dissertation CTU in Prague (2009, in Czech).

15. J. Př́ihoda, P. Louda, Mathematical modelling of turbulent flow, CTU in Prague (2007, in Czech)

16. J. Šimonek, K. Kozel, J. Trefilík, Proceedings: Topical Problems of Fluid Mechanics 2007, 169 (2007) 


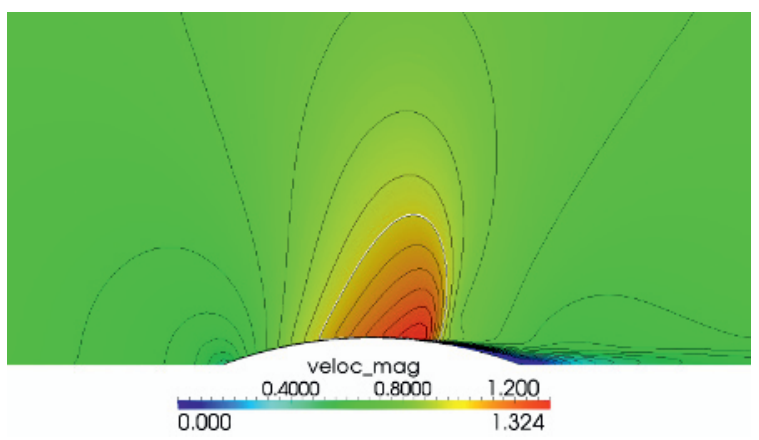

Fig. 2. Symmetric DEIW configuration, viscous flow, $M_{\infty}=0.775, \alpha=0^{\circ}, R e=10^{7}, k-\omega$ model (SST variant), Mach number isolines

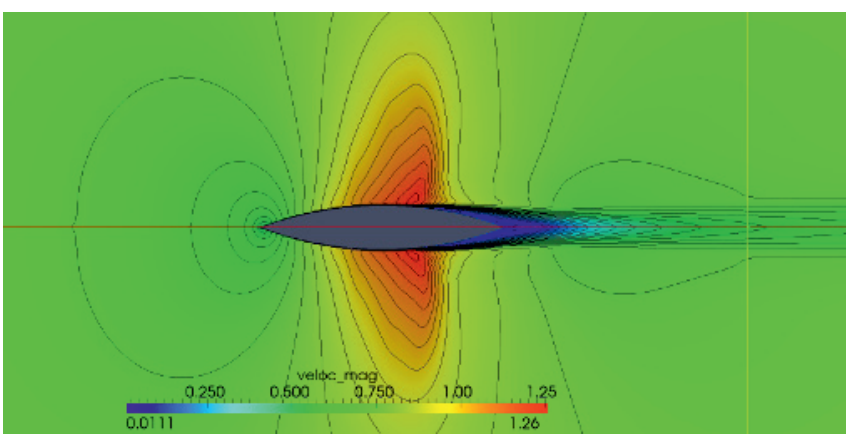

Fig. 3. Symmetric DEIW configuration, viscous flow, $M_{\infty}=0.775, \alpha=0^{\circ}, R e=10^{6}, k-\omega$ model (TNT variant), Mach number isolines

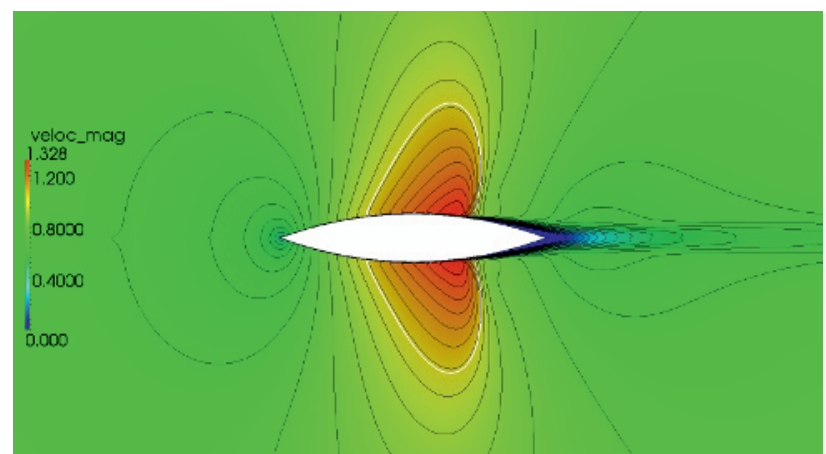

Fig. 4. Symmetric DEIW configuration, viscous flow, $M_{\infty}=0.775, \alpha=0^{\circ}, R e=10^{7}, k-\omega$ model (SST variant), Mach number isolines

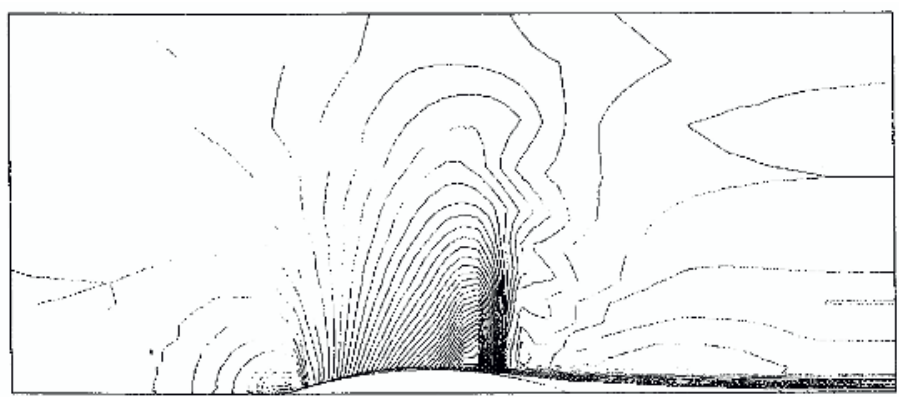

Fig. 5. DEIW configuration reference result, viscous flow, $M_{\infty}=0.775, \alpha=0^{\circ}, R e=10^{7}$, simple mixing length model, Mach number isolines 